\title{
A Serious Game for Predicting the Risk of Developmental Dyslexia in Pre-readers Children
}

\author{
Ombretta Gaggi, Giorgia Galiazzo, Claudio Palazzi \\ Dept. of Mathematics \\ University of Padua \\ Padua, Italy \\ Email: \{gaggi, cpalazzi\}@math.unipd.it
}

\author{
Andrea Facoetti, Sandro Franceschini \\ Developmental and Cognitive Neuroscience Lab \\ Dept. of General Psychology \\ University of Padua \\ Padua, Italy
}

Email: andreafacoetti@unipd.it, sandrofranceschini@gmail.com

\begin{abstract}
Dyslexia is one of the main cognitive disabilities affecting the reading acquisition. As it may interfere with educational opportunities even in presence of adequate intelligence, it is of crucial importance to obtain early diagnosis and help affected children as soon as possible. Generally, dyslexia is diagnosed not earlier than primary school as difficulties in reading is used as first indicator. Yet, being able to detect and treat this problem even in preschool years would ensure better chances to limit its impact and help the child's future reading ability. To this aim, we discuss here a series of serious games we designed and tested to train specific skills that have been proven to be effective against dyslexia.
\end{abstract}

\section{INTRODUCTION}

Dyslexia, also known as reading disorder, is a learning disability affecting the ability to process written, and sometimes even spoken, language. A person with dyslexia has difficulty with the alphabet, reading comprehension, writing and spelling in spite of adequate intelligence, exposure, and cultural opportunity. Dyslexia is often a cause of school failure and significantly affects the child's education, as well as being an important risk factor for the development of more complex psychopathological disorders (e. g., behavior disorder, anxiety, and depression).

This disorder accounts for approximately $80 \%$ of all specific learning disabilities, and is a crucial obstacle to the development of a educated, competent and competitive society [1].

One of the main challenges with dyslexia is to obtain an early diagnosis in very young children, in order to help the affected child as soon as possible, i.e., during the first years of the primary school. A study conducted on preschool children that have a familiar risk for dyslexia proves that visual spatial attention and speech segmentation are both impaired in those subjects [2]. A 3-years longitudinal study have demonstrated the multi-factorial hypothesis of dyslexia, suggesting that not only auditory-phonological deficits, but also visual spatial attention are causally implicated in dyslexia [3]. Thereby, the combined use of investigation tools in early detection can result highly effective for these children. Furthermore, the rehabilitation methods which stimulate both the phonological skills and the visual-spatial attention are proved to be the most effective in children already diagnosed with dyslexia [4] [5]. Training these skills before the child begins to learn reading, can help his/her future reading ability.
In this context, our goal is the creation of a series of serious games which could help training these skills by just playing [6], [7], [8], [9]. The idea is to hide exercises to train both visual-spatial attention and speech segmentation underneath a game, so children can improve their learning capability while playing. The serious game paradigm is therefore used to maintain user attention and to reduce the drop out from treatment: it is difficult that a child stop playing if the game is engaging, whereas it is certainly easier to have a child not performing an exercise when it is considered boring.

This paper presents our serious games created to test in different ways abilities which are usually impaired in subjects with dyslexia. In particular we primarily test the development of functions which are deeply related to the children's future reading skills, i.e., the eye-hand coordination, visual search ability, rapid identification of visual and acoustic inputs and the capability to associate visual and auditory stimuli. Our approach aims at implicitly training these capabilities through games and is a novel approach in the treatment of dyslexia.

To allow an effective treatment, each child needs to perform the same set of exercises for about 20-30 minutes per day, every day for at least one month: a training of this duration may be sufficient to develop the individual skills that will be useful to learn to read. For these reason, a second crucial goal we reached was to ensure that the created games were engaging so as to transform an annoyed patient into an excited player.

\section{EMPLOYMENT SCENARIOS}

Before designing the application, we performed an evaluation of the employment scenarios to identify adequate devices and development technologies. Indeed, our serious games will be used in different contexts. When used as a treatment, the child patient has to frequently interact with it for a certain period of time to maximize its benefits; we hence need a tool that can be used even at home. On the other hand, the initial approach with the tool for assessing potential dyslexia in child patient are performed in a specific structure such as the children psychologist's studio or even at school.

Computers and Internet connectivity are typically present in domestic environments, but the same cannot be confidently said for psychologists' studios and schools. Moreover, while 
at home, the application and the device could be used by a single child, whereas in structures they are probably going to be shared, in turn, by several children.

Having children playing with our serious games at home passes through the possibility, for their families, to have a device with adequate features and compatibility for running the software. To this aim, the application has to be portable over different platforms so as to let families use any device they may already have at home without the cost of purchasing a new one. As it is well known, nowadays the Web is a programmable environment that ensures the widest portability.

Summarizing the aforementioned requirements, the devised serious games have to ensure the widest portability regardless of the device (e.g., PC, tablet, etc.) and of being online or offline. Finally, the age of considered children is a crucial aspect. Indeed, considered preschool children are too young to perform a confident diagnosis, which will have to wait till the second year of primary school, when children start reading and writing fluently ${ }^{1}$. At preschool age, we can evaluate only a "dyslexia risk", which represents an important indicator since sooner treatments could produce better results and even reduce the disorder; yet, an identified dyslexia risk does not necessarily imply the future development of the disorder. Given this lack of confidence in identifying preschool children with dyslexia potentiality and the potential benefits of the treatment, it may be advisable to administer the developed serious games to all preschool children.

\section{Developed Games}

In this section we describe the most representative games we have developed to assess and treat dyslexia in preschool children. Each of these games is designed to train specific skills by exploiting visual cue stimuli and distractors. Cue stimuli are used to have the player focusing on the target, whereas distractors generate opposite effect. The use of these stimuli affects the players' performance, especially in younger ones such as preschoolers; we have hence designed our games to increase the use of cue stimuli with players having gaming difficulties, while the occurrence of distractors increases with more skilled players [10].

\section{A. Game \#1: Paths}

Paths is a game we created to train user's capability to rapidly discriminate among images in the fovea region and in the surrounding ocular area. The fovea constitutes the central area of the retina and it is characterized by the sharpest, most acute visual acuity; specifically, it determines a neat vision of the specific point or object we are looking at, while the contours are perceived blurred. The game invites the child to track some obliged paths to reach a final point. These paths consist of a number of open circles (similar to the letter $C$ ) oriented in the four directions left-right-up-down, as shown in Figure 1.

\footnotetext{
${ }^{1}$ This study was conducted in Italy, where, due to the fact that the Italian language can be defined a semi-transparent language, children start reading fluently during the second year of the primary school.
}

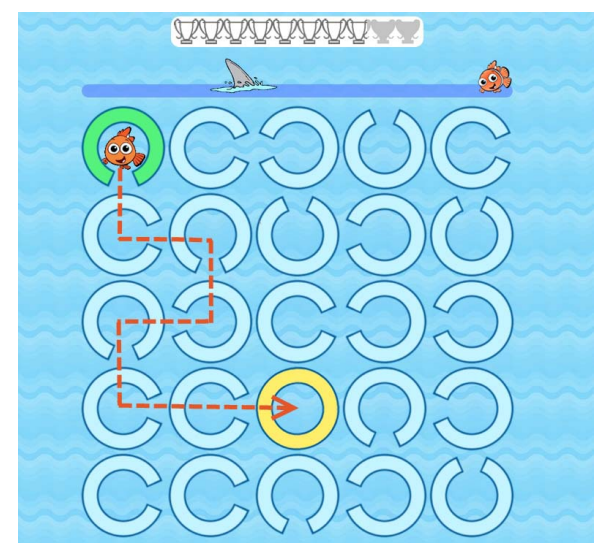

Fig. 1. Paths game.

This exercise involves a visual search, which implies the ability to process some stimuli; this process is the result of the child's ability to anchor, focus and disanchor. The crowding effect, meaning the difficulty in the visual recognition of a target surrounded by other visual elements, plays a defying role in the child performance. To successfully finish the game, the child must improve his/her ability to analyze each element between the starting one and the final target, so as to plan the his/her moves.

The participant starts at the green open circle and has to reach the yellow closed circle within a pre-defined time lapse to win the game. To reach the final target, the player has to move from one open circle to the other considering its orientation. Each open circle is opened on only one side giving the only direction of allowed move into the next open circle. At the very beginning the player is helped by a cue element, a visual stimulus pointing to the right direction which is then removed when the player acquires some familiarity with the game. The game difficulty increases according to the increasing ability of the child, gradually reducing the size of the opening in the circles.

Every day five matches will be played. At the successful conclusion of the previous match, a new, more difficult path will be presented. Otherwise, the same path will be proposed again to facilitate the child in the task.

\section{B. Game \#2: Fence Letters}

We designed and implemented a game, named Fence Letters, to train the child to focus on local stimuli, ignoring the picture which is a large letter with partially dashed boundaries. The subject will be asked to close lines in the letter, as he/she is eliminating holes in a fence before fishes swimming inside can escape (see Figure 2).

Each time the child identifies a hole in the fence and closes it, the complete outline of the fence (the large letter) is highlighted and a sound is generated to reinforce an implicit association grapheme-phoneme [11]. As the skills of the player improves, the difficulty level of the game increases by adding new fishes and augmenting their speed. This process helps the development of a split attention in the player as he/she 


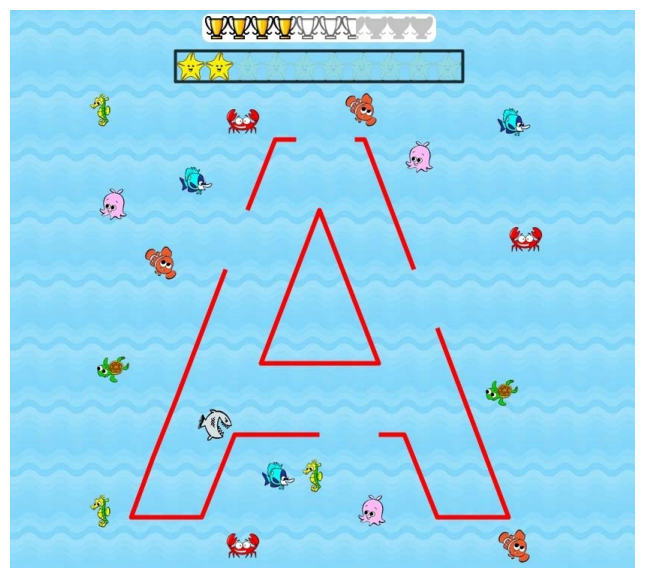

Fig. 2. Fence letters game.

has to pay attention to the movements of two or more stimuli (i.e., the fishes trying to escape from the fence letter), which requires both wide and perimeter-specific attentional focus. Furthermore, for the duration of the game, the letter remains a marginal detail for the child: there will be no interaction directly related to its meaning, but only related to its presence as an object of the game.

\section{Game \#3: Wizards}

In 1980, Dr. Paula Tallal hypothesized that at the basis of language disorders, and partially of dyslexia, there was a defect in the brain zone that processes rapid auditory stimuli [12]. When this happens, instead of being processed correctly, acustic stimuli are overlapped and mixed with other sounds. It is also argued that reading deficits mainly derives from an erroneous categorization of sounds forming words, caused by impaired capability to discriminate among acoustic features of different sounds and phonemes.

In a Temporal Order Judgment (TOJ) task, the player has to identify which one between two auditory stimuli is emitted first. Indeed, it is well known that subjects suffering from dyslexia have difficulties in the rapid processing of the stimuli. The TOJ task is specifically designed to tests and trains this ability, by generating sounds that the subject has to discriminate depending on their emission order.

To this aim we created a TOJ-based game named Wizards, where the temporal discrimination of sounds is presented as a fun challenge, with two wizards competing in magic. Specifically, a letter is assigned to each wizard who will pronounce it at a certain point to cast a spell. The first wizard pronouncing its letter will transform some crabs into tiny monsters. Then, the player has to indicate (through the touch screen) which wizard cast the spell by identifying the pronounced letter so as to cancel the spell and re-transform the tiny monsters into crabs.

The two letters associated to the two wizards that are presented to the subject are chosen in couples among those known as hard to be distinguished such as, for instance, $P$ and $B$. Each phoneme lasts for $150 \mathrm{~ms}$, which is the minimum amount of

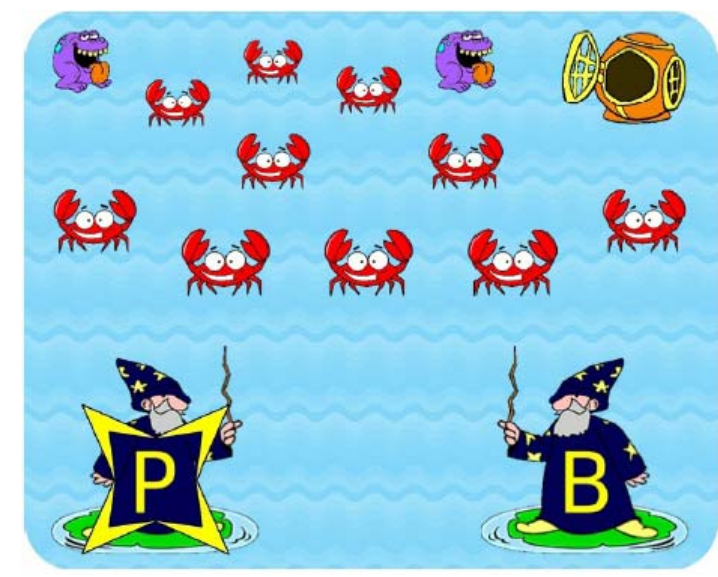

Fig. 3. Wizards game; a cue is visible on the leftmost wizard.

time necessary to identify the corresponding letter [13]. The time interval between the end of the sound of a letter and the beginning of the next one has a variable duration that depends on the child's ability. Initially, beside the phoneme there will be also a visual stimulus (a cue, as shown in Figure 3) associated to the letter that will help the player in understanding the task. The cue will be eliminated after few rounds of the game.

\section{System Architecture And DeVelopment}

As already discussed in Section II the proposed serious games must be accessible everywhere, both on-line and offline, in order to adapt themselves to the biggest number of different situations in which a user can play. Moreover, the particular target users, preschool children, requires particular attention to the interaction paradigm which is used.

Therefore, the design of the system architecture must take into account two different issues:

- the usability of each game, considering the particular target users and

- the portability of the overall system.

Some studies [14] have proposed the touch interfaces as a suitable interaction paradigm for very young children, even of 2 years old. Their tests showed that this interaction paradigm is considered very natural [15]. Indeed, children can interact with touch screen devices (e.g. tablets) without asking them to learn using standard peripherals such as a keyboard (preschool children do not know letters) or a mouse: they simply interact with the device by touching the screen with a finger in the same way they would touch an object they like. For this reason, touch interfaces are an excellent candidate for interfaces of applications targeted for children, since they require users to learn only few simple gestures to play with their fingers on the screen.

In order to address portability, all considered games must be playable on any device. For this reason we implemented our application as a rich internet application (RIA). Each game is a web application accessible through any browser: successful tests have been carried out on Microsoft Internet 
Explorer $^{2}$, Mozilla FireFox ${ }^{3}$, Google Chrome ${ }^{4}$ and Apple Safari $^{5}$, both on the Apple and Microsoft main operating systems. Thanks to HTML5 features cache manifest and local storage, at the first access, the browser downloads all it needs to play the game. After this first step, all considered games can be played even in absence of Internet connectivity, which is required only to send to a server data collected during the exercises at the end of the session. In this way, we also minimize the network usage for communication between server and client.

Moreover, several game sessions can be played without sending data: this operation is required only when the local storage is full. In this case, the game is blocked and cannot be played until the data are sent to the server and the local storage is emptied. Therefore, the server has two main tasks:

- to provide the games and

- to collect data about the exercises performed by each user, in order to check the progress of the training activity.

In realizing a cross-platforms application, audio files are provided both in MP3 format, supported by Chrome, Internet Explorer and Safari, and using ogg/vorbis encoding, supported also by Firefox and Opera. For the same reason, we used Web Storage instead of Web SQL Database, since the first one is already supported by a larger set of browsers.

The server is implemented on a LAMP (Linux, Apache, MySql, PHP) architecture: it collects data on a MySql database that can be queried by the administrator, or the children psychologist, through a set of web pages that retrieve data using the PHP language.

In order to address usability, we tested the game with a touch device that can hence be easily brought into a school. First, we tested our system with a touch screen connected with a computer. This test resulted into a better usability for the involved children, but touch screens are not usually available in many schools and they are not completely portable for their size, weight and need to plug in for electricity. Therefore, a set of tests have been performed using two tablets, an Apple iPhone and an Android Asus TFT101. These tests revealed that both the devices were initially not able to run the games, in particular they did not support the simultaneous playing of more than one audio file.

To solve this problem, we needed two applications, one specific for each platform, and a technology capable of carrying out a conversion from a web application to a native application, i.e., the PhoneGap framework [16].

\section{USER STUDY}

Our system has been tested in several ways to verify its robustness, correctness and usability. To what concerns usability, two sets of users tested our games.

\footnotetext{
${ }^{2}$ version 9 or higher. IE version 9 does not support to play the game off-line.

${ }^{3}$ version 3.6 or higher.

${ }^{4}$ version 10 or higher.

5 version 5 or higher.
}

A first, smaller, group of children, between 3 and 6 years old, has tested the system during all the phases of its development. The goal of this first set of tests was to evaluate the interaction paradigm both with children that have never used a computer before these experiments and children that have some (very little) knowledge about standard input devices, such as mouse and keyboard. Moreover, we want to test whether the children have fun with our games or they get bored after a while. These tests were very useful to design a correct interface, easy to use, and enjoyable for children: they confirmed that even preschool children are able to use touch interface in a very natural way and that 6 years old children can also quickly learn to use standard input devices to play with our games.

Moreover, these tests have shown that employed audio files and the graphic design are suitable for the target children: the games are able to engage children even when played several times. Since the treatment requires children to play every day with our games for a period of, on average, half an hour, this result is particularly important to lower the percentage of drop out from therapy.

Since target users are preschool children between 5 and 6 years old, it is particularly important to use images which do not hurt or frighten them. As an example, we initially use a shark pin getting closer and closer to a baby to show the amount of elapsed time in the Paths game: when the pin (and the shark underneath) reaches the baby, the game is over. Younger children (in particular females) reported to be scared by this animation, but as soon as we changed the image of a baby with the image of a red fish (see the time-bar on the top-most side of Figure 1), children did not complain about it anymore.

At the end of the development, a second group was involved in the experiment. This second group considered users closer to the target audience and included 24 children of the last year of a kindergarten in Padua, Italy.

Each child played one complete session of the treatment, i.e. he/she plays a match with all games one after the other. We would have preferred to repeat the game session more than once for each child; unfortunately, this was not possible due to the limited time granted by the school. Before starting the games, we gave very few instructions to each child individually to explain the goals of each game. To this end we used some printed images of the system interface.

The test campaign lasted for two days and the tests were carried out using two iPads. Clearly, we cannot be sure that in a real scenario all children will have this kind of device available, but at home children will have plenty of time to learn how to use a computer with keyboard and mouse so as to resort to more popular devices (even if tablets are nowadays becoming more and more common in homes). Performed tests also involved a psychologist, who had previously evaluated the risk of dyslexia of all children.

We have asked a feedback from our testers and the outcome reveals that the children like the games and consider them easy to play. In Figure 4 and Figure 5, "P" denotes the Paths game, 


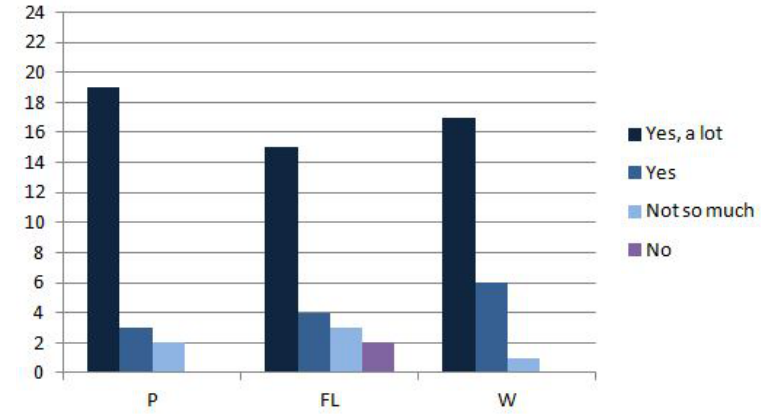

Fig. 4. Answers to the question: "Do you like the game?"

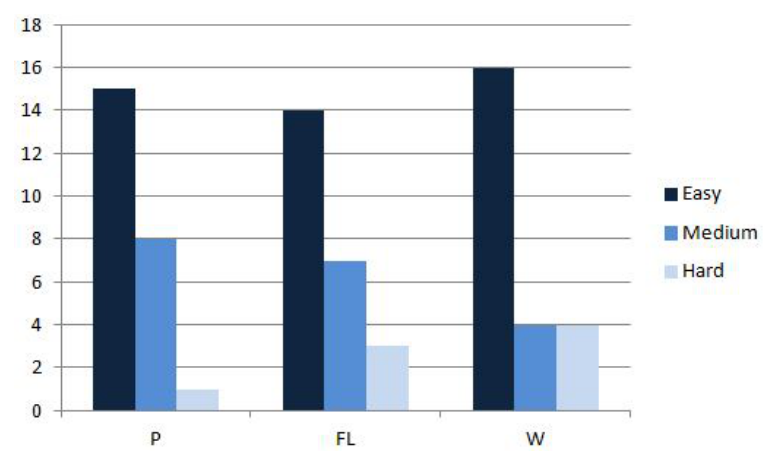

Fig. 5. Answers to the question: "Is the game difficult to play?"

"FL" denotes the Fence Letters game and "W" represents the Wizards game.

At the end of each game session, we asked to every child whether he/she liked the game. Figure 4 details the answers to this question: the child can choose between four possibilities ("Yes, a lot", "Yes", "Not so much" and "No"). On average, $70 \%$ of the children chose the first option, only two children declared that they did not like at all the Fence Letters game, whereas the other games reported "Not so much" as worst answer and provided only by very few children.

We asked to the children to assess the difficulty level of each single game. The outcome is depicted in Figure 5. On average, $62 \%$ of the children considered our games easy to play. We must note here that our main goal was not to develop games which should necessarily be considered easy by all children. In fact, we mainly aimed at developing games that could be used as a training treatment for particular skills, with a daily frequency. With too simple games, there would be no skill training. Therefore, each game must be properly calibrated in order to be neither too difficult nor too easy, otherwise the risk of drop out from treatment arises. Our developed games resulted to be not too complex for preschool children; at the same time, they are able to train the skills of interest by adapting the difficulty according to the skills of the user.

\section{CONCLUSION}

In this paper we have presented a system composed by various serious games designed for predicting the risk of developing dyslexia in very young children. Once these subjects have been identified, our system can also be used to train phonological skills and the visual-spatial attention, which are usually impaired in these children, in order to improve their future reading ability.

We performed a user study to prove that our serious games can be played by preschool children and that they also represent an engaging activity. Consequently, we can state that our serious games can be used as daily treatment with a low probability of drop out.

We are currently testing, with preliminary encouraging results, whether our serious games allow to discriminate between children with a high risk of dyslexia and other children, based on the players' performance.

\section{ACKNOWLEDGMENT}

Partial financial support for this work is provided by MIUR/PRIN ALTER-NET and the UNIPD/PRAT Web Squared projects.

\section{REFERENCES}

[1] T. Vidyasagar and K. Pammer, "Dyslexia: A deficit in visuo-spatial attention, not in phonological processing," Trends in Cognitive Sciences, vol. 14, no. 2, pp. 57-63, 2010.

[2] A. Facoetti, N. Corradi, M. Ruffino, S. Gori, and M. Zorzi, "Visual spatial attention and speech segmentation are both impaired in preschoolers at familiar risk for developmental dyslexia," Dyslexia, no. 16, pp. 226239, 2010.

[3] S. Franceschini, S.Gori, M.Ruffino, K. Pedrolli, and A. Facoetti, "A causal link between visual spatial attention and reading acquisition," Current Biology, vol. 22, pp. 1-6, 2012.

[4] J. D. Gabrieli, "Dyslexia: a new synergy between education and cognitive neuroscience," Science, vol. 325, pp. 280-283, 2009.

[5] A. Facoetti, M. L. Lorusso, P. Paganoni, C. Umiltà, and G. G. Mascetti, "The role of visuospatial attention in developmental dyslexia: evidence from a rehabilitation study." Brain Res. Cogn. Brain Res., vol. 15, pp. 154-164, 2003.

[6] C. E. Palazzi, M. Roccetti, and G. Marfia, "Realizing the unexploited potential of games on serious challenges," ACM Computers in Entertainment, vol. 8, no. 4, pp. 4-7, 2010.

[7] D. Deponti, D. Maggiorini, and C. E. Palazzi, "Smartphones physiatric serious game," in Proc. of the IEEE Intl. Conf. on Serious Games and Applications for Health (SeGAH 2011), November 2011, pp. 647-656.

[8] L. A. Ripamonti and D. Maggiorini, "Learning in virtual worlds: A new path for supporting cognitive impaired children," in Proc. of the 14th Intl. Conf. on Human-Computer Interaction (HCI 2011), July 2011, pp. $462-471$.

[9] M. Roccetti, G. Marfia, and A. Semeraro, "Playing into the wild: A gesture-based interface for gaming in public spaces," Journal of Visual Communication and Image Representation, Elsevier, vol. 23, no. 3, pp. 426-440, 2012.

[10] M. W. G. Dye, C. S. Green, and D. Bavelier, "The development of attention skills in action video game players," Neuropsychologia, no. 47, pp. 1780-1789, 2009.

[11] Y. Sasaki, J. E. Nanez, and T. Watanabe, "Advances in visual perceptual learning and plasticity," Nature Reviews Neuroscience, no. 11, pp. 5360, 2010.

[12] P. Tallal, "Auditory temporal perception, phonetic, and reading disabilities in children," Brain and Language, no. 9, pp. 182-198, 1980.

[13] U. Goswami, "A temporal sampling framework for developmental dyslexia," Trends in Cognitive Sciences, no. 15, pp. 3-10, 2011.

[14] A. D. Bortoli and O. Gaggi, "PlayWithEyes: A new way to test children eyes," in Proc. of the IEEE Intl. Conf. on Serious Games and Applications for Health (SeGAH 2011), November 2011, pp. 190-193.

[15] C. Forlines, D. Wigdor, C. Shen, and R. Balakrishnan, "Direct-touch vs. mouse input for tabletop displays," in Proc. of ACM CHI 2007 Conf. on Human Factors in Computing Systems, May 2007, pp. 647-656.

[16] Adobe Systems, "Phone Gap Framework," http://phonegap.com/, 2012. 